CAHIERS DE

NARRATOLOGIE
Cahiers de Narratologie

Analyse et théorie narratives

15 | 2008

Récits et genres historiques

\title{
Dictature, témoignage, histoire : paroles de victimes et de bourreaux dans les littératures argentine et chilienne (1983-2002)
}

José García-Romeu

\section{(2) OpenEdition}

Journals

Édition électronique

URL : http://journals.openedition.org/narratologie/796

DOI : $10.4000 /$ narratologie.796

ISSN : 1765-307X

Éditeur

LIRCES

Référence électronique

José García-Romeu, « Dictature, témoignage, histoire : paroles de victimes et de bourreaux dans les littératures argentine et chilienne (1983-2002) », Cahiers de Narratologie [En ligne], 15 | 2008, mis en ligne le 14 décembre 2008, consulté le 15 novembre 2019. URL : http://journals.openedition.org/ narratologie/796; DOI : 10.4000/narratologie.796

Ce document a été généré automatiquement le 15 novembre 2019.

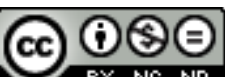

Cahiers de Narratologie - Analyse et théorie narratives est mis à disposition selon les termes de la licence Creative Commons Attribution - Pas d'Utilisation Commerciale - Pas de Modification 4.0 International. 


\title{
Dictature, témoignage, histoire : paroles de victimes et de bourreaux dans les littératures argentine et chilienne (1983-2002)
}

\author{
José García-Romeu
}

En 1964, la dictature de Castello Branco fait du Brésil le laboratoire d'un modèle politique baptisé "Doctrine de Sécurité Nationale» qui s'appliquera ensuite en Argentine (1966-1974 et 1976-1983), en Uruguay (1971-1985), au Chili (1973-1989) ainsi qu'au Paraguay où la dictature du général Alfredo Stroessner (1954-1989) s'était constituée au départ autour d'une idéologie populiste et sociale. Cette doctrine actualise dans le contexte de la guerre froide la pensée nationale catholique qui avait inspiré dès les années vingt la droite autoritaire de nombreux pays de culture ibérique. À partir de l'expérience brésilienne, ces droites militaristes adaptent à la réalité américaine, en lien avec les Etats-Unis, les théories de lutte antisubversive élaborées par l'Armée française pendant les guerres d'Indochine et d'Algérie, exposées en 1961 par le colonel Trinquier dans La guerre moderne (1961). Elles possèdent également un théoricien autochtone: le général brésilien Golbery do Couto e Silva, auteur de Planejamento estratégico (1955) et Geopolitica do Brasil (1967) qui trouvera un illustre épigone en Augusto Pinochet (Geopolítica, 1974). La Doctrine de Sécurité Nationale détermine l'existence d'un conflit universel qui oppose la tradition catholique, le libéralisme économique et une hiérarchisation autoritaire de la société, à un marxisme diabolisé dont on recule les limites à l'ensemble des courants réformistes de sorte que la moindre expression du relativisme moral ou du scepticisme religieux est suspecte de trahir une compulsion révolutionnaire ${ }^{1}$. L'ampleur et la nature insidieuse du conflit exigent des actions radicales telles que le recours massif aux détentions arbitraires, aux disparitions ${ }^{2}$, à la torture, à l'exil forcé... l'objectif étant d'instaurer l'uniformisation idéologique de la société. 
2 Le terrorisme d'État menace plus particulièrement les militants syndicaux et politiques, les journalistes critiques, les membres d'associations d'entraide sociale, les avocats engagés dans la défense des victimes de la répression et les intellectuels anticonformistes. Elle oblige ces derniers à élaborer de nouvelles formes de contrediscours alors que la protestation traditionnelle, sous l'effet de la censure et de la contrainte physique, est réduite au silence. À ce propos, outre les écrivains qui échappent par fortune aux persécutions arbitraires et parviennent à développer des stratégies de contournement ${ }^{3}$, nous pouvons évoquer les auteurs ayant subi la réclusion et l'exil, qui exorcisent leur expérience en s'interrogeant sur leur survie d'expatriés ${ }^{4}$, et ceux, témoins précoces plus que victimes directes des dictatures, qui porteront sur ces années un regard historique investi par les questions de justice et de faute. Un partage générationnelle se manifeste entre les premiers, qui s'efforcent de surmonter des épreuves singulièrement graves et les seconds, plus jeunes, qui réélaborent des mémoires vieilles d'un quart de siècle afin de constituer les preuves à charge contre les responsables de la terreur. D'une génération à l'autre, les auteurs abandonnent le statut de victime pour celui de juge et leur travail de deuil, qui visait à recouvrer un nouvel équilibre individuel, pour le réquisitoire public et historique.

Pour illustrer cette évolution, nous évoquerons un roman et un recueil poétique argentins, Libro de navíos y borrascas de Daniel Moyano ${ }^{5}$ et Interrupciones 2 de Juan Gelman $^{6}$, ainsi que deux romans plus récents, Nocturno de Chile du chilien Roberto Bolaño ${ }^{7}$ et Dos veces junio de l'argentin Martín Kohan ${ }^{8}$.

I. L'exil

4 L'expérience de l'exil, qui domine l'écriture de Gelman et de Moyano, servira de point de départ à notre réflexion en permettant de contextualiser dans le débat littéraire l'une des impulsions centrales de ces écritures : l'interrogation autour des culpabilités réciproques, celles des assassins et de leurs complices, celles des exilés ayant abandonné leurs camarades et leur langue maternelle, celles enfin d'auteurs ayant renoncé à une expression entièrement libre afin de survivre dans leur pays.

5 Nous ne reviendrons pas sur la querelle qui déchira à ce sujet l'intelligentsia argentine ${ }^{9}$ sinon pour rappeler l'opposition entre intellectuels adhérant à l'idée d'un public international sensible à une parole sincère, non contrainte par la censure, et intellectuels, attachés à l'expression d'inquiétudes locales n'ayant de sens que pour des lecteurs nationaux. Pour les premiers (Julio Cortázar, Juan Carlos Martini...), seul l'exil garantissait la liberté de parole indispensable à l'écriture alors que les seconds (Luis Gregorich, Liliana Heker...) y voyaient une grave démission vis-à-vis de la réalité, de la langue maternelle et des compatriotes demeurés au pays.

Objectivement, il faut admettre que la nature de l'exil dont furent victimes Gelman et Moyano ne correspond pas à l'idée défendue par Liliana Heker d'un exil égoïste excité par la richesse éditoriale étrangère. Le départ de Moyano fut la condition nécessaire à sa libération de prison, obtenue par des pressions internationales. Pour Gelman, elle fut la condition de sa survie. Membre d'une organisation armée de l'extrême gauche péroniste, Montoneros, il vit tomber en effet de nombreux camarades, dont les écrivains Francisco Urondo et Rodolfo Walsh.

II. Première génération1. Daniel Moyano (1930-1992) : Libro de navíos y borrascas (1983)

7 Catholique progressiste engagé auprès des déshérités, Moyano fut arrêté en 1976. Libéré, il se rendit à Madrid et retranscrivit les effets de la répression dans $E l$ vuelo del tigre (1981). L'invention d'un espace (le village de Hualacato) qui rend archétypiques 
certains traits de La Rioja, sa région d'adoption, la métaphorisation de la réalité politique sur le ton d'une fable où l'ensemble du pays est rapporté à une maison familiale... tout cela manifeste la volonté de surmonter, par l'allégorie et le merveilleux, l'échec de l'engagement politique qui avait détourné beaucoup d'écrivains du réalisme appliqué aux représentations des faits sociaux. Rappelons que cette démarche avait suscité deux textes novateurs, ayant eu un grand impact sur de nombreux auteurs, El fiord (1969) d'Osvaldo Lamborghini et El frasquito (1973) de Luis Gusmán. Ces récits représentaient la violence politique à travers le sadisme domestique et furent interdits par la dictature. D'une certaine façon, El vuelo del tigre recourut à un jeu de métaphorisation de la terreur assez similaire, encore qu'en termes moins crus et moins libidinaux.

Dans Libro de navíos y borrascas, Moyano rompt avec ses œuvres antérieures, d'abord en abandonnant le sujet de l'exil intérieur pour celui de l'exil extérieur, ensuite en explorant - comme s'il cherchait à rétablir par la référence immédiate une langue maternelle menacée par l'exil - une écriture moins métaphorique où les déterminations spatiales et temporelles se précisent. Le collage, le pastiche, le recours à diverses modalités génériques (pièce pour marionnettes, récits courts, paroles de tango)... contribuent à établir un complexe faisceau de stratégies narratives coordonnées tandis que l'élaboration autobiographique prise en charge par la voix du personnage Rolando participe à composer un récit cathartique ayant pour but de sublimer un vrai traumatisme subi par l'auteur.

Rolando raconte donc son voyage d'exil en compagnie d'autres infortunés. Le navire qui les conduit porte un nom au symbolisme transparent, Cristóforo Colombo:

Le voyage à bord du Cristóforo Colombo entre les ports de Buenos Aires et de Barcelone de sept cents Argentins, Chiliens et Uruguayens victimes des trois coups d'État des années soixante-dix, établit un pont allégorique entre deux catégories spatio-temporelles qui sous-tendent toujours les œuvres de la littérature de l'exil. Ici c'est le bateau, le premier refuge avant la terre d'asile, avançant sur la mer, base mouvante de l'imaginaire étalée entre deux là-bas. Le premier, celui du pays que l'on quitte, fait partie des terres de la mémoire. Le deuxième, encore inconnu, est un pays irréel et il peut seulement être exploré par l'imagination. ${ }^{10}$

10 Le retour sur soi et sur le passé se substitue au discours contestataire et prospectif des décennies précédentes, le futur n'exprimant plus que l'inquiétude curieuse envers la terre d'accueil. Alors que les traumatismes de la répression rendent caducs les programmes destinés à changer le monde à venir, le narrateur et ses camarades d'exil examinent leur expérience en l'inscrivant dans de vastes généalogies familiales ou dans l'histoire plus ou moins lointaine : dans de vastes généalogies car Rolando présente son périple non comme un exil, mais comme le retour à la patrie de ces ancêtres espagnols, atténuant ainsi, par un pis-aller astucieux, la blessure de l'expatriation; dans l'histoire grâce à une pièce pour marionnettes où certains des exilés vont retracer l'exécution de Dorrego par le général Lavalle (1828), épisode de l'histoire argentine considérée comme point de départ des luttes ayant abouti au coup d'État de 1976 que l'on pourrait qualifier, en suivant Ricœur, d'«héritage de la violence fondatrice » partageant la mémoire entre exécration et célébration, entre regret humilié des vaincus et glorification commémorative des vainqueurs ${ }^{11}$.

11 Ainsi la grande histoire est ramenée aux proportions d'un théâtre de marionnettes qui renverse - en soutenant une interprétation proche de celle du révisionnisme de gauche incarné par David Viñas (1929) et sa revue Contorno (1953-1959) - la figure du général 
Lavalle, héros de l'histoire officielle. L'œuvre de marionnettes peut également s'interpréter comme un contre-pied au Romance de la muerte de Juan Lavalle, texte tragique de l'écrivain Ernesto Sábato dont un enregistrement, auquel participèrent des figures musicales aussi célèbres qu'Ernesto Falú et Mercedes Sosa, fut largement diffusé dans les années soixante ${ }^{12}$. Travaillant dans un registre populaire similaire à celui de Sábato, registre dont ils préservent cependant le caractère carnavalesque et subversif, les artistes du Libro de navíos y borrascas contredisent à la fois l'image empesée des manuels scolaires et le penchant tragique du grand écrivain consacré. Le meurtre de Dorrego ne se lit plus comme une fatalité incompréhensible mais comme un abject crime idéologique.

Quant à la généalogie familiale, souvenons-nous que dans El vuelo del tigre déjà, Nabu, l'oppresseur, interdisait aux Aballay de regarder les photos de famille afin d'empêcher l'insertion du présent dans une longue relation où tel ascendant pouvait être érigé en symbole de résistance et suggérer les vertus d'une histoire alternative. Tandis que la grande histoire officielle construit des artifices héroïques afin de justifier le rôle des militaires, il ne reste aux opposants que les lambeaux de confuses diégèses familiales où les moutons noirs retrouvent a posteriori, dans les limites de cette perspective domestique, une position centrale permettant de réinterpréter le devenir historique. Aussi les textes de l'époque évoquent-ils souvent, à l'instar des romans de Moyano, des récits familiaux tronqués et erratiques fort éloignés des sagas à la Robert Merle et qui opposent des contre-modèles aux grands récits officiels (Ricardo Piglia, Respiración artificial, 1980 ; Marta Lynch, La penúltima versión de la Colorada Villanueva, 1979...). Nous observerons que Gelman esquissa également, sans trop le développer cependant, cette sorte de rappel généalogique.

2. Juan Gelman (1930) : Interrupciones 2 (1988)

Comme l'indique Interrupciones 2, Gelman porte l'exil dans son histoire familiale :

Mi padre vino a América con una mano atrás y otra adelante, para tener bien alto el pantalón. Yo vine a Europa con una [sic.] alma atrás y otra adelante, para tener bien alto el pantalón. Hay diferencias, sin embargo : él fue a quedarse, yo vine para volver. ${ }^{13}$

Gelman est en effet le premier fils argentin d'une famille de juifs ukrainiens débarquée en Argentine en 1928 afin de fuir les persécutions politiques. Pétri par l'entretien laïc de ses racines juives et par la sensibilité révolutionnaire de ses parents, il contribua dès les années cinquante à diffuser un discours poétique engagé dans la vie populaire. En 1975, alors que se mettait en place la terreur d'État, il dut s'exiler tandis que son fils et sa belle-fille disparaissaient. En 1979, il rompit avec la direction des Montoneros qui venaient d'engager les quelques militants rescapés dans une délirante offensive finale. Depuis lors, il n'a cessé d'être loué comme l'un des poètes les plus populaires d'Argentine. Son talent sera internationalement consacré en 2007 par l'attribution du prestigieux prix Cervantes.

Interrupciones 2 représente un aboutissement de la rénovation ayant rompu dans les années 50, sous les auspices de César Fernández Moreno (1919-1985), avec la génération dite de 40, dénomination commode qui regroupait arbitrairement - sous prétexte de consigner la relève des ultraïstes (Borges, Girondo...) par de jeunes créateurs - aussi bien des surréalistes (Olga Orozco, Enrique Molina...) que des poètes élégiaques traditionnalistes (Roberto Paine, Juan Rodolfo Wilcock...). Juan Gelman, Leónidas Lamborghini, Francisco Urondo, Noe Jitrik, Edgar Bayley... introduisent alors dans la 
poésie argentine une veine nationale, critique et populaire. Beaucoup d'entre eux collaboreront à la revue Zona (fondée en 1963) et exprimeront un engagement politique entre marxisme et péronisme. Leur démarche contestataire pourrait être rapprochée de celle du groupe réuni autour de David Viñas dans la revue Contorno.

Interrupciones 2 associe une grande diversité de thèmes et de figures entremêlés: biographie familiale et élégie de l'exil, réécriture de textes anciens et entretien de la mémoire... Cette mémoire se décline selon deux registres : celui rapporté à l'expérience historique récente, qui assume souvent le devoir de dresser l'éloge funèbre des camarades tombés, non sans exprimer un diffus sentiment de culpabilité pour avoir survécu, et celui d'une mémoire ancienne rapportée aux racines de lointaines généalogies familiales et culturelles juives. Cette hétérogénéité manifeste sans doute un long et difficile travail de deuil.

(a section la plus ancienne, « Bajo la lluvia " (écrite en 1980) Gelman y indique que préserver le sentiment d'étrangeté propre à l'exil est indispensable à la conservation de la langue maternelle (texte $V, p .16)$. Dans le même sens, le texte VI confirme certaines des thèses de Gregorich et de Heker dont nous parlions plus haut, puisqu'il est dit que l'exil impose une solitude sans langue, brise la communication et dessèche l'écriture. Gelman distingue alors une forme d'incommunicabilité tournée vers le futur et le présent, applicable à l'indicible tel que la littérature l'a toujours sondée, d'une forme orientée vers le passé, provoquée par le naufrage en terre étrangère (texte VII, p. 18). Poursuivant sur ce mode dialectique, Gelman oppose également l'image du sang qui coule à la fermeté du sol :

Estoy soñando con la vía del Corso y el Jorge con la rue de Mouffetard. Calles soñadas por nosotros, de espaldas a nosotros, que nunca nos sabrán. Las pisamos de día y de noche, pasamos por ellas, nos desangramos contra ellas, sueños de piedra que reconoce otros pies. ${ }^{14}$

L'exil paraît un écoulement qui échappe à la stabilité, dans un pays d'asile inconnaissable. Il est souffrance dans un enfer où l'on subit une de ces tortures éternelles imaginées par la pensée grecque: répétition, douleur et frustration provoquées par l'aigle qui dévore le foie ou par le rocher qui roule au pied de la colline, toujours. C'est ainsi que l'exilé Juan et l'exilé Jorge saignent jour et nuit, en parcourant sans fin des rues étrangères. L'exil est également souffrance dans une autre sorte d'enfer, où s'organise une file d'attente en vue du jugement dernier et dans lequel les âmes, mêlées dans un même désastre, ont perdu leur identité originelle :

Hacemos cola ante el país, al descampado, llueve, se alzan lenguas de fuego que lamen a los santos, las calaveras pasan pajareando, senos de una mujer arrastran cielo, la cola de 14.000 kilómetros viborea, hierven los argenguayos, urulenos, chilentinos, paraguanos, están tirando de la noche sudamericana, rechinan de almas en silencio, su verdadero trabajo. ${ }^{15}$

19 Par cette voie, Gelman retrouve des sensibilités et des représentations anciennes, judéo-chrétiennes ou grecques. La corrélation de l'histoire récente à des figures issues des racines profondes de la culture occidentale apporte ainsi un premier apaisement à l'effroi et à la catastrophe. Le travail de deuil peut alors commencer.

20 C'est encore en recourant au passé culturel que Gelman parvient à résoudre définitivement le traumatisme et à recouvrer l'énergie nécessaire pour poursuivre une lutte aux accents patriotiques. Car malgré l'expérience douloureuse qui l'a expulsé du pays pour faire à rebours le voyage du père, Gelman ne cesse de crier son amour pour la patrie. Il est pourtant héritier de ces immigrés auxquels certains intellectuels 
nationalistes, dont l'écrivain Leopoldo Lugones ${ }^{16}$, prêtèrent dans les années 20 un rôle néfaste : non catholiques, parlant mal le castillan, gagnés à des idéologies suspectes, ils étaient accusés d'altérer gravement l'identité nationale. Dans Interrupciones 2, Gelman associe les effets de la mémoire historique, ceux de l'expérience individuelle et enfin ceux de l'exploration littéraire, pour démonter le discours nationaliste, source d'inspiration de la dictature de 1976.

21 La mémoire historique contredit d'abord l'idée selon laquelle l'Argentine est un pays exclusivement européen issu d'un processus colonial positif, car la colonisation, dont l'exilé trouve en Europe les effets contradictoires, ne fut pas l'aventure de peuplement épique que put y voir l'extrême droite argentine :

En vía del Corso y Bulmish huelo de pronto a taino devorado por perros andaluces,

a orejas de ona mutilado, a azteca deshaciéndose en el lago de Tenochtitlán, a

inquita roto en Potosí, a querandí, araucano, congo, carabalí, esclavizados, masacrados [...].

Olés [Europa] a doble humanidad, la que asesina, la que es asesinada. ${ }^{17}$

L'expérience individuelle ensuite inscrit Gelman, fils de juif ukrainien, dans une argentinité que le poème exprime de façon insistante et qui pousse l'écrivain à résister à l'intégration en terre étrangère, laquelle représenterait une défaite finale et l'oubli des camarades morts au pays :

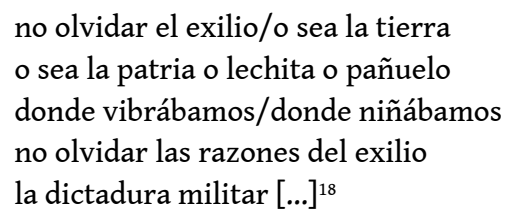

L'exploration littéraire enfin se manifeste dans «Com/posiciones»(1984-1985) à travers la réécriture des manuscrits de la mer morte, d'épisodes bibliques et de poésies juives et arabes médiévales qui permettent d'exhumer les origines hétérodoxes de Gelman ainsi que celles de cette Espagne des trois religions, anéantie jadis par le catholicisme impérial auquel l'idéologie nationaliste s'identifie. Argentinisés par la parole du poète contemporain, ces textes mettent au jour les influences multiples qui imprègnent l'identité nationale et répliquent au projet autoritaire d'épuration culturelle et idéologique.

Les poèmes de "Com/posiciones ", les plus récents du recueil, révèlent un dépassement de l'élégie et du repli commémoratif propres aux autres sections. Gelman y produit une proposition identitaire forte et parvient à relancer efficacement la bataille idéologique contre la pensée autoritaire, attestant de la sorte avoir recouvré toutes ses forces.

3. Première conclusion

La fracture entre l'avant et l'après de la répression constitue une catastrophe. Celle-ci n'est lisible que grâce au travail de mémoire qui va progressivement l'objectiver en fait historique, intégrable à une suite événementielle plus profonde (généalogique et/ou nationale), moyennant quoi la catastrophe devient compréhensible et narrable. Ce travail de mémoire concourt ainsi à résoudre une expérience douloureuse, laquelle est en grande partie indicible ${ }^{19}$ en raison de la souffrance extrême infligée aux victimes et de la dégradation de leurs capacités à communiquer. Concernant ce dernier point, vital pour les systèmes de communication que sont la littérature et le témoignage, il est notoire que la dictature affecte les échanges verbaux de graves symptômes pathologiques en favorisant la censure, l'isolement carcéral, le secret inhérent à la clandestinité des opposants, la parole confessionnelle arrachée sous la torture, 
l'altération de la langue maternelle en exil, l'imprégnation de tout discours par le discours autoritaire agoraphobe... Paul Ricœur écrit :

[...] le crédit accordé à la parole d'autrui fait du monde social un monde intersubjectivement partagé. Ce partage est la composante majeure de ce qu'on peut appeler «sens commun». C'est lui qui est durement affecté lorsque des institutions politiques corrompues instaurent un climat de surveillance mutuelle, de délation, où les pratiques mensongères sapent à la base la confiance dans le langage..$^{20}$

Reconstruction thérapeutique élevé contre ces maladies du discours et inséré dans un vaste réseau de causes et d'effets historiques, le témoignage personnel, qu'il soit factuel et lyrique (Gelman) ou fictionnel et narratif (Moyano), fait fonction de travail de deuil et permet à la victime réelle (l'écrivain) de dépasser le trauma individuel grâce à un discours explicatif pouvant être assorti à autrui. À ce propos, rappelons que la pratique contemporaine d'une sorte de micro-histoire du passé récent (nécessairement assez récent pour pouvoir compter des témoins vivants) basée sur la promotion d'individus communs et des victimes de désastres historiques, consiste effectivement en l'expression d'un témoignage personnel destiné à être inscrit dans un recueil, une collection polyphonique qui attesterait de l'articulation entre expérience individuelle et fait social.

Il faut se demander maintenant comment on glisse, de ce travail de mémoire soutenu par le témoignage individuel de la victime, au réquisitoire méticuleux contre les bourreaux qui exige de ceux-ci qu'ils retrouvent la mémoire exacte de leurs fautes. C'est encore Ricœur qui indique une piste possible :

[...] que manque-t-il au travail de mémoire et au travail de deuil pour s'égaler au devoir de mémoire ? [...] c'est l'élément impératif [...]. Plus précisément, ce qui fait encore défaut, c'est le double aspect du devoir, comme s'imposant du dehors au désir et comme exerçant une contrainte ressentie objectivement comme obligation. Or, où ces deux traits se trouvent-ils réunis [...] sinon dans l'idée de justice [...]? C'est la justice qui, extrayant des souvenirs traumatisants leur valeur exemplaire, retourne la mémoire en projet; et c'est ce même projet de justice qui donne au devoir de mémoire la forme du futur et de l'impératif. ${ }^{21}$

Pourtant, alors que Ricœur fait entendre que cet impératif naît du "souvenir traumatisant », propre donc aux victimes ou à ceux qui par compassion ou par lien de descendance leur sont proches, Bolaño et Kohan montrent que c'est la parole et la mémoire des bourreaux et de leurs complices qui permettra d'établir un «devoir » de mémoire, impossible à contredire dès lors que la vérité dont il se souvient ne sera plus exclusivement assurée par la parole solitaire des victimes.

III. Seconde génération1. Roberto Bolaño (1953-2003) : Nocturno de Chile (2000) Aunque vivo desde hace más de veinte años en Europa, mi única nacionalidad es la chilena, lo que no es ningún obstáculo para que me sienta profundamente español y latinoamericano. En mi vida he vivido en tres países : Chile, México y España. ${ }^{22}$

29 A l'inverse des écrivains argentins précédents qui expriment un grand attachement à leur patrie, le chilien Roberto Bolaño a davantage vécu à l'étranger, et cela dès son enfance. En 1973, quelques jours après le coup d'État, ce jeune homme de 20 ans arriva au Chili après un long périple depuis le Mexique. Il en fut rapidement expulsé pour avoir fait mine de résister à la dictature lors d'un épisode dont lui-même parlera avec humour et détachement bien qu'il eût risqué sa vie. Il poursuit dès lors une carrière d'écrivain étrangère à la réalité littéraire et éditoriale chilienne qui le conduira du Mexique en Espagne. Il fréquentera un monde intellectuel cosmopolite, représenté 
entre autres par l'argentin Rodrigo Fresán ou le mexicain Mario Santiago. Par ailleurs, Bolaño appartient à une génération qui a intégré l'échec des grands discours révolutionnaires : s'il ne renonce pas à exprimer ses idées politiques, il n'abrite plus l'espoir de changer le monde à court terme et se satisfait de changer la littérature.

Nous nous sommes efforcés de décrire naguère ${ }^{23}$ comment, dans Nocturno de Chile, le prêtre Sebastián Urrutia Lacroix, membre de l'opus Dei et critique littéraire, produisait au terme de sa vie une sorte de confession hypocrite visant à camoufler les fautes passées, et en particulier la complicité envers un régime criminel. Nous voudrions étudier cette fois-ci l'inculpation des pratiques d'oubli, de dissimulation et de refoulement sur lesquelles fut construite une démocratie névrosée, concédée par des militaires chiliens n'ayant pas subi, à l'inverse des argentins, une déroute politique définitive. L'exercice de Bolaño est particulièrement sensible car il fait parler, non pas les victimes qui réclament le droit à la parole, mais un complice des bourreaux, c'est-àdire justement un de ceux qui participèrent dès l'origine à censurer et à taire le récit des crimes répressifs. Le renversement est complet puisque l'auteur ne transfert pas seulement la narration de la victime au bourreau, mais également de celui qui réclame le droit à témoigner à celui qui refuse l'obligation de le faire. Dès lors qu'elle assume cette mission délicate, la fiction ne peut avoir recours qu'au discours intérieur, le seul que le coupable puisse assumer, et le seul que l'écrivain - qui n'est pas, lui, un tortionnaire - puisse efficacement médiatiser. L'exposition des fautes et des justifications étant cantonnée à la conscience individuelle et soustraite au débat public contradictoire, la justice ne devrait pouvoir être exercée que par une instance morale intérieure au narrateur. Cependant, bien que sa formation chrétienne lui permette d'entrevoir la mesure de sa culpabilité, le discours intérieur d'Urrutia Lacroix a justement pour fin d'éluder une confession sincère. Bolaño utilise donc une astuce littéraire en décrivant comment le prêtre est hanté dans son délire par le regard critique de celui qu'il nomme lui-même " el joven envejecido ", dont on ignore s'il s'agit d'un vrai personnage ou d'un double réprobateur d'Urrutia Lacroix. En effet, ce « joven envejecido » est à la fois dépeint comme un jeune écrivain inscrit dans une rupture idéologique et générationnelle avec Urrutia (p. 24), ou comme la voix de la conscience intérieure (p. 149). La vraisemblance de cette contradiction est soutenue par le fait que le prêtre est emporté par le délire de l'agonie.

31 Compte tenu de l'absence, dans la société chilienne, d'institutions officielles et collectives ayant pris efficacement en charge l'acte de justice ${ }^{24}$, l'écrivain chilien pourvoie cette justice d'un corps littéraire. Invention de Bolaño attribuée à la fantaisie délirante d'Urrutia Lacroix, ce "joven envejecido » est un personnage doublement fictionnel (et donc doublement littéraire). Il est par ailleurs le procureur qui dénonce non seulement la complicité politique mais également la complicité esthétique de ce critique conformiste et conservateur. La faute d'Urrutia Lacroix apparait dès lors à travers deux activités corrélées qui répondent à même mobile normatif: l'activité politique et l'activité de critique littéraire.

Puisque la justice institutionnelle n'est pas en mesure de juger Urrutia Lacroix, Bolaño élève donc un tribunal littéraire particulièrement efficace car sa capacité créatrice agit comme un sérum de vérité en ouvrant les vannes d'une confession qui finit par se dire à contrecœur, et par se trahir dans ses justifications embarrassées. La nature fantasmatique de cette sorte d'instance juridique démontre cependant la précarité du 
procédé dans une société incapable d'assumer publiquement le procès des anciens oppresseurs.

2. Martín Kohan (1967) : Dos veces junio (2002)

Engagé dans une approche savante de l'écriture, aussi bien en tant que créateur que critique littéraire, Kohan enseigne la littérature à l'Université de Buenos Aires et participe activement à de nombreux débats, parfois polémiques, dans les revues culturelles argentines actuellement en vue. Dans des romans à résonance historique, il a fouillé les mythes argentins fondateurs en recourant aux grandes figures de San Martín et d'Esteban Echeverría (El informe, 1997; Los cautivos, 2000). Dos veces junio poursuit ce travail autour de la mémoire collective, rapportée cette fois-ci à la dictature de 1976. L'auteur consacre une première partie du récit au mois de juin 1978 en décrivant pour une part des événements liés à la répression et pour une autre la défaite de l'Argentine contre l'Italie pendant la coupe du monde de football, qui se jouait alors en Argentine. Dans la partie finale, plus courte, il replace en juin 1982, après la défaite de la guerre des Malouines contre la Grande Bretagne et pendant une autre coupe du monde, certains des personnages rencontrés précédemment. À travers un récit fragmenté en séquences rarement plus longues qu'une page, Kohan évoque la déficiente conscience politique des classes moyennes et la bienséance idéologique imposée par des oppresseurs aux faux airs de gentlemen. La narration principale est produite par une jeune recrue, chauffeur d'un médecin militaire, qui exprime en toute bonne conscience une confiance aveugle envers le devoir patriotique, la hiérarchie de classe et la hiérarchie de grade, confiance qui lui fait trouver tout naturel qu'on puisse s'interroger sur l'âge à partir duquel on peut torturer un enfant. Ce récit autobiographique, largement dominant, reçoit comme contrepoint un ensemble de récits entrelacés à la troisième personne et adoptant le point de vue des victimes ou celui de médecins tortionnaires. Sont ainsi évoqués les traitements subis par une femme qui accouche sans soins dans une prison clandestine, ou l'humiliation d'une jeune fille violée par cinq soldats. Par ailleurs, de ridicules observations méthodiques sur divers sujets (le football, les pèse-personnes...) s'alignent sur un même plan d'expertise technique, dont toute dimension morale est absente, à des considérations sur la torture. Ce jeu de contraste et de confusion met en évidence l'efficacité d'une bureaucratie répressive reposant sur une chaîne de commandement bien rôdée.

Ici, une trentaine d'année après $E l$ frasquito de Luis Gusmán et El fiord d'Osvaldo Lamborghini, la violence sexuelle sert de nouveau à représenter la violence politique. Cette fois-ci cependant, les deux violences ne sont plus liées par la métaphore qui permettait de médiatiser l'une à travers la perversion omnipotente de l'autre. Le récit se réfère au contraire à l'horrible réalité telle qu'elle fut rendue publique par Nunca más en $1984^{25}$, le sadisme sexuel étant pour le bourreau un moyen privilégié d'exercer sur des prisonniers en voie de disparition sa toute puissance politique. Contredisant encore la métaphorisation, les violeurs et tortionnaires sont directement désignés en tant que militaires bénéficiant d'une totale impunité.

Kohan innove par ailleurs en emboîtant le témoignage de la victime dans celui du complice, le narrateur principal rapportant comment la jeune mère torturée dont nous parlions plus haut sollicitera son aide à travers la porte de sa cellule. Elle lui demande de contacter un avocat et raconte en détail les traitements qu'elle aura subis pour justifier cette démarche légale. Le narrateur extradiégétique restitue le fait sans retranscrire le témoignage de la femme, qu'il écoute jusqu'au bout sans cesser pourtant 
de lui répéter : «Callate de una vez [...] hija de puta [...]. » Nous apprenons par ailleurs, par la voix du médecin qui suit l'état de santé de cette femme, qu'elle n'a pas cédé à la torture et qu'elle n'a dénoncé aucun complice. La nature des événements répressifs placent témoignages et narrations dans un jeu de relations complexes : la victime, qui n'a rien avoué à ses bourreaux, témoigne auprès d'un de leurs complices avec l'espoir que celui-ci contactera un avocat dans un pays où la justice, incapable de poursuivre les criminels et de les interroger, est réduite à enregistrer la découverte de corps mutilés, impossibles à identifier, des victimes de la répression. Dans Dos veces junio, une des nouvelles données par le journal que lit le narrateur principal en juin 1982 atteste d'ailleurs cette réalité du cadavre enfoui, découvert ici à l'occasion d'un chantier.

Ainsi, le système des témoignages établi par Kohan est bien plus complexe et plus sombre que dans les textes que nous avons étudiés précédemment: d'une part, à l'inverse de ce qui se passe dans les œuvres de Gelman et de Moyano, la parole de la victime ne permet aucune sublimation post-traumatique, puisque elle est plongée dans l'horreur et sur le point de mourir; d'autre part, à la différence de Nocturno de Chile, le statut confessionnel de la narration du complice ne se vérifie pas, car l'esprit du narrateur n'est pas même effleuré par la question de sa propre culpabilité. Au contraire, le témoignage de la femme torturée semble répéter pour le narrateur la rencontre de la nuit précédente avec une prostituée, et le «callate, hija de puta » qu'il prononce à plusieurs reprises réduit leur relation à un jeu obscène et pervers dans lequel la femme joue sa vie et celle de ses compagnons de captivité tandis que l'homme assouvit son plaisir sadique ${ }^{26}$.

37 N'imaginons pas toutefois qu'à l'inverse de Bolaño, qui construit la confession du narrateur à des fins d'inculpation, Kohan se contente de rapporter un témoignage neutralisé par la bonne conscience du narrateur dans le seul but d'effrayer le lecteur par tant d'aveuglément moral. L'exposition de l'impunité des bourreaux, de la non assistance à personne en danger, de l'inconscience de la culpabilité et du mal, participe également à désigner la faute. Mais en l'absence d'un « joven envejecido ", il échoit ici au lecteur d'assumer le réquisitoire contre le narrateur. Dès lors, le texte n'a de sens que s'il revient à considérer comme destinataire, non pas le lecteur savant capable de déchiffrer les énigmes métaphoriques d'une littérature autoréférentielle, mais la communauté nationale des lecteurs. Kohan retrouve ainsi la confiance en une littérature engagée capable de peser sur le débat public et à laquelle avaient en partie renoncé Moyano, Gelman et Bolaño.

\section{Seconde conclusion}

Bolaño et Kohan engagent la critique de la dictature et du discours autoritaire sur des voies fort éloignées de celles prises par Moyano et Gelman. Cette différence se lit essentiellement dans un glissement radical du témoignage de la victime - identifiable en termes plus ou moins directs à l'auteur - à la confession ou explication du bourreau et de ses complices - adversaires exécrés par l'écrivain. Du témoignage de soi au témoignage de l'autre, on passe d'une volonté de retrouver sa place dans le monde, suite à l'expulsion soufferte, à un travail fictionnel plus ambigu qui prétend, comme pièce à charge dans une sorte de réquisitoire à venir, exposer les mobiles du meurtrier. Tandis que Moyano et Gelman recouraient à la littérature pour sublimer leur traumatisme, Bolaño et Kohan en usent à l'inverse pour plonger dans le cloaque de consciences malades et criminelles. Par ailleurs, ces écrivains innovent en développant cette parole du coupable à travers une expression ample et riche, là où des romanciers 
plus âgés s'étaient contentés de la parodier et de la caricaturer. Souvenons-nous en effet comment, afin de combattre la censure et l'hypertrophie du discours autoritaire, Osvaldo Soriano (Cuarteles de invierno, 1982), Manuel Puig (El beso de la mujer araña, 1976), Humberto Costantini (Dioses, hombrecitos y policías, 1979) et Ricardo Piglia (Respiración artificial, 1980 et La ciudad ausente, 1992) prêtaient l'essentiel de leur écriture à une parole subversive rendue muette par la répression, et limitaient la retranscription du discours autoritaire à ses représentations les plus schématiques (rapports de police grotesques ou déclamations primaires et grandiloquentes). À l'inverse, les romans de Bolaño et de Kohan gravent directement, de façon particulièrement minutieuse, la parole des oppresseurs en faisant paraître, respectivement, leur tartufferie ou leur effroyable expertise de tortionnaires.

\section{NOTES}

1. Cf. CONADEP, Nunca más. Informe de la Comisión Nacional sobre la Desaparición de Personas, «Capítulo V. El respaldo doctrinario de la represión », Eudeba, Buenos Aires, 1984, p. 473-476 ; Catherine Durandin, «L'idéologie de la sécurité nationale au Brésil », Problèmes d'Amérique Latine, XLIV, 17 juin 1977, p. 5-18.

2. La détention non déclarée, suivie du meurtre et ensuite de la dissimulation du corps, fut une des méthodes les plus employées à l'époque. Sur cette question, nous nous permettons de renvoyer à notre article : « Disparus : les procédures de l'élimination politique en Amérique Latine. Traitement littéraire d'un concept paradoxal ", Figures de la disparition dans le monde hispanique et latino-américain, Revue Babel, $\mathrm{n}^{\circ} 13$, Francisco Albizú Labbé (éd.), Université du Sud, 2006, p. 107-120.

3. Pour ne plus revenir sur ces stratégies du contournement, citons les exemples de Ricardo Piglia qui camoufle le contre-discours au moyen d'une critique littéraire sophistiquée (Respiración artificial, 1980) ; de José Pablo Feinmann qui choisit le roman policier (Ni el tiro del final, 1981) pour représenter la violence politique au moyen du crime crapuleux ; d'Ernesto Schoó (El baile de los guerreros, 1979) qui situe cette violence dans un cadre historique afin de simuler une certaine distance avec la réalité présente ; d'Ana María Shua qui prend l'espace hospitalier comme métaphore d'un pays infantilisé et opprimé (Soy paciente, 1980).

4. Cf. Olver Gilberto de Léon (éd.), Novela y exilio, Montevideo, Signos, 1989.

5. Daniel Moyano, Libro de navíos y borrascas, Buenos Aires, Editorial Legasa, 1983.

6. Juan Gelman, Interrupciones 2, Buenos Aires, Seix Barral, 2006 (1ère éd. 1988).

7. Roberto Bolaño, Nocturno de Chile, Barcelona, Anagrama, 2000.

8. Martín Kohan, Dos veces junio, Buenos Aires, Editorial Sudamericana, 2002.

9. Cf. José García-Romeu, « La littérature argentine et les frontières de l'exil : conflits et solutions dans l'espace fictionnel », À propos de la frontière. Liminaires-Passages interculturels italo-ibériques, LEIA Vol. 2-2003, Bernard Fouques (éd.), Bern, Peter Lang, 2002, p. 87-101.

10. Sara Bonnardel, La répression et l'exil dans les romans de Daniel Moyano, Document de travail nº 7 - 1990, G.I.R.D.A.L., Université de Bordeaux III, Talence, p. 61. 
11. Paul Ricœur, La mémoire, l'histoire, l'oubli, Paris, éditions du Seuil, 2000, p. 99.

12. Romance de la muerte de Juan Lavalle, Phonogram S.A.I.C., 1964 ; Ernesto Sabato : textes et récitatif ; Eduardo Falú : composition, chant et guitare ; Mercedes Sosa : chant ; Francisco Javier Ocampo : direction des chœurs.

13. Op. cit., p. 23.

14. Op. cit., p. 19.

15. Op. cit., p. 20.

16. À propos de l'idéologie nationaliste qui inspira le discours autoritaire argentin, $c f$. José García-Romeu, « Chapitre I : le discours autoritaire », Dictature et littérature en Argentine, Paris, L'Harmattan, 2006, p. 17-52.

17. Op. cit., p. 38.

18. Op. cit., p. 16.

19. Depuis Auschwitz, on n'ignore pas le caractère indicible de l'exaction de masse et du génocide.

20. Op. cit., p. 207.

21. Op. cit., p. 107.

22. « Bolaño por Bolaño », La escritura como tauromaquia, Celina Manzini (éd.), Buenos Aires, Corregidor, 2002, p. 202.

23. José García-Romeu, « Los trucos del confesante o el suplicio de la memoria : Nocturno de Chile de Roberto Bolaño ", Ecritures des dictatures, écriture de la mémoire : Roberto Bolaño, Juan Gelman, Carmen Vásquez, Ernesto Mächler Tobar, Porfirio Mamaní Macedo (coord.), Paris, Indigo-Université de Picardie-Jules Verne, 2007, p. 71-84.

24. Les obstacles auxquels a été confronté le juge Guzmán indiquent qu'au Chili, à l'inverse de l'Argentine, les conditions politiques ne sont pas encore réunies pour permettre un jugement des agents de la répression.

25. $C f$. note 1 .

26. La résistance de cette femme et son refus d'avouer pose en termes de thématique littéraire le problème du témoignage arraché par la torture et de l'héroïsation de la victime. Roberto Bolaño dans Estrella distante (1996) et Ricardo Piglia dans Respiración artificial (1980) et La ciudad ausente (1992) évoquent ces questions.

\section{RÉSUMÉS}

Les dictatures militaires qui gouvernèrent le Chili et l'Argentine dans les années soixante-dix et quatre-vingt eurent un impact considérable sur les écrivains. Nous étudierons comment certains textes littéraires abordent la question à travers une reconstruction de la mémoire et de l'histoire, nationale ou familiale, en recourant au témoignage des victimes ou des bourreaux. Parfois l'auteur médiatise son expérience personnelle, d'autres fois il fabrique une fiction complexe et ambiguë. Cette étude nous permettra de distinguer à ce propos deux générations, la première soucieuse de résoudre les traumatismes propres (Daniel Moyano, Libro de navíos y borrascas, 1983 ; Juan Gelman, Interrupciones 2, 1988), la seconde engagée dans un réquisitoire envers les responsables de la répression (Roberto Bolaño, Nocturno de Chile, 2000 ; Martín Kohan, Dos veces junio, 2002). 
Las dictaduras militares que gobernaron en Chile y en Argentina durante los años setenta y ochenta tuvieron un impacto importante en los escritores. Estudiaremos cómo algunos textos literarios contemplan el problema mediante una reconstrucción de la memoria y de la historia, nacional o familiar, recurriendo al testimonio de las víctimas y de los verdugos. A veces, el autor representa su experiencia personal, otras fabrica una ficción compleja y ambigua. Este estudio nos ayudará a distinguir dos generaciones, la primera preocupada en resolver los traumas propios (Daniel Moyano, Libro de navíos y borrascas, 1983 ; Juan Gelman, Interrupciones 2, 1988), la segunda comprometida en acusar a los responsables de la represión (Roberto Bolaño, Nocturno de Chile, 2000 ; Martín Kohan, Dos veces junio, 2002).

INDEX

Mots-clés : Daniel Moyano, dictature, Juan Gelman, Martín Kohan, mémoire, Roberto Bolaño Index chronologique : XXe - XXIe siècles 